\title{
The effect of transcutaneous electrical nerve stimulation (TENS) and cryotherapy in the relief of pain during labor
}

\author{
Mariana Meurer Regis ${ }^{(1)}$, Gesilani Júlia da Silva Honório ${ }^{(2)}$, Keyla Mara dos Santos ${ }^{(1)}$, Soraia Cristina Tonon da Luz ${ }^{(2)}$, \\ Clarissa Medeiros da Luz ${ }^{(2)}$, Thuane da Roza ${ }^{(1)}$
}

\begin{abstract}
Background: The delivery pain is an unpleasant experience which may generate some insecurity for the woman. Thus, some methods of analgesia need to be studied in order to generate greater comfort for the woman during labor. Objective: to evaluate the effect of transcutaneous electrical nerve stimulation (TENS) and cryotherapy (CRYO) on labor pain. Method/Design: quasi experimental study, randomized sample in two groups: TENS and CRYO. Pregnant women were selected in the Carmela Dutra Maternity (Florianópolis (SC), Brazil) according to inclusion criteria: women which are pregnant for the first time, age (18-40 years) and initial cervical dilatation of $4-5 \mathrm{~cm}$. The Visual Analogue Scale (VAS) was used to evaluate pain before and after each intervention. Results: 24 parturient women were studied (TENS $=11, C R Y O=13)$. In the TENS group, there was a significant result in pain relief after the first application $(p=0.002)$. VAS was reduced from 8 to 5.5 , but not significant. In the CRYO group there was reduction of pain during the first application $(p=0.008)$, and in the second there was an increase in pain $(p=0.008)$. There was a significant difference between the techniques in the evaluation performed before the second application, with a lower value attributed to the CRYO group $(p=0.034)$. Conclusions: CRYO and TENS were effective in relieving pain during the first phase of the initial phase of labor. However, during the treatment, in the parturient women studied, there was maintenance of pain response by the CRYO group and decrease in the TENS group without reduction significant.

Keywords: Labor; TENS; Cryotherapy; Pain; Physiotherapy.
\end{abstract}

\section{INTRODUCTION}

Pregnancy and childbirth are described as, for all women, a process accompanied by profound physical and psychosocial changes, and for many of them occur as an unpleasant experience due to the pain experienced when the time of delivery. ${ }^{(1)}$

Childbirth care has several difficulties due to the public policies implemented in the health sector in Brazil. It is known despite the incentives and recommendations of Ministry of Health of Brazil in supporting the normal birth, indeed the incidence of cesarean deliveries has increased. (2) Davim and colleagues $^{(2)}$ state that one of the main factors influencing the high incidence of cesarean births are, among others, the fear of pain during labor and delivery.

The pain of childbirth is an ancient human experience, but it is still an inherent reality. For most women, the pain of childbirth is seen as a rather unpleasant experience. Although pain is a reality, it may identify different behaviors in relation to the experience. ${ }^{(3)}$

The International Association for the Study of Pain defines pain as a sensory experience, emotionally unpleasant. It is surrounded by unpleasant, subjective feelings, each individual relates to pain according to their previous experience and it is, in a manner, an emotional experience. ${ }^{(2)}$

In addition to the sense of pain, the concern of how to relieve it is also present in the care of laboring women. Pain is a multidimensional and subjective experience that may be sized by professionals only through its manifestations. ${ }^{(4)}$ The pain of labor may be alleviated or abolished since it may interfere with the physiological processes, and it can produce various changes in the cardiovascular, respiratory and gastrointestinal systems, and cause psychological, physical and biochemical disorders. ${ }^{(5)}$ The intense pain affects the contractility of the uterus and its blood flow, which prejudice the delivery development, also commonly may occur apnea by the parturient, resulting in decreased uterine blood flow, which may lead to fetal hypoxemia. ${ }^{(5)}$

Health professionals have shown interest and concern in the alternatives methods which may provide relief to these women. ${ }^{(6,7)}$ They also concern about the effects that an anesthesia might have on the mother and baby. Thus, these

Corresponding Author: Keyla Mara dos Santos. Rua Pascoal Simone, 358, Coqueiros, Florianópolis, SC, Brazil, CEP: 88080-350. E-mail: keylasantosfisio@gmail.com 'Universidade do Estado de Santa Catarina (UDESC), Florianópolis, SC, Brazil

Full list of author information is available at the end of the article.

Financial support: The authors declare no financial support. 
factors should encourage professionals to research new alternative methods and disseminate such procedures, as well as to prove their effectiveness in that type of population. Some non-pharmacological alternatives are available to minimize the pain during labor, such as massages, chiropractic, acupuncture, homeopathy, among others. ${ }^{(6,7)}$

Currently, a safe, free of side effects and risk alternative for the mother and fetus is Transcutaneous Electrical Nerve Stimulation (TENS), which through electrodes placed on the skin, conduct electrical stimuli, causing analgesia. ${ }^{(6,7)}$

Another alternative and analgesic method is the ice therapeutic application, the Cryotherapy (CRYO). ${ }^{(8,9)}$ This technique reduces a gradual manner the transmission of sensory impulses to decrease the speed of its driving, thereby reducing the activation of nociceptors, it occurs the decrease of pain transmission, as well as the reduced neural metabolism. ${ }^{(10)}$

The effectiveness of TENS pain relief has been researched and really supported by many studies, ${ }^{(11,13)}$ although the number of studies proving its effectiveness in relieving pain during labor is still small. ${ }^{(14)}$ On the other hand, articles relating cryotherapy to labor are scarce.

Aiming to improve the humanization of delivery, Miquelutti and colleagues ${ }^{(15)}$ indicates the necessity to identify evidencebased practices which may contribute to the relief of pain during labor and to the mother and child welfare. Thus, this study is justified by the fact that pain, as already mentioned, is an unpleasant sensation and an uncomfortable experience, often reported as unbearable.

Therefore, this study aims to evaluate the effect of the TENS and CRYO techniques in relieving pain during labor.

\section{METHOD}

This research is characterized as analytical quasiexperimental type, it aims to determine through the intervention which technique is more effective in relieving the pain of women during labor. ${ }^{(16)}$

The study was approved by the Carmela Dutra Maternity Ethics Committee, Florianópolis - Santa Catarina (SC), Brazil under protocol 0042.0.233.000-10. All participants were informed about the study procedures and after agreeing to participate, they signed the informed consent form. A sample of 24 parturient hospitalized at the Carmela Dutra Maternity Hospital was involved from January to April 2011. The selection occurred through daily visits to the obstetric hospital unit.

As inclusion criteria, the mother should be primiparous, aged between 18 and 40 years and present initial cervix dilation 4-5 cm. High-risk pregnancies, twin pregnancies were excluded. The choice of primiparous mothers is justified by the fact that the pain tends to be minimized and better supported as they occur over two deliveries, so in the studied cases, this experience would be unprecedented. (17)
The sample was randomized into two groups, TENS Group with a total of 11 participants and the CRYO group with 13 participants. Before the intervention was filled in the registration form with personal data and gestational history, but also the mother responded to the data of visual analogue scale (VAS) pain and indicated the site with the aid d drawing body McGill pain questionnaire. ${ }^{(18)}$

The TENS group received technical intervention by placing adhesive type electrodes 4 cross-art in the paravertebral region between T10 and L1, because it is more effective to reduce pain frames present in the lower abdomen and lower back. (19) The apparatus used was TENS 993, Quark brand, applied to a frequency of $150 \mathrm{~Hz}$, pulse duration of $80 \mu \mathrm{s}$ and the intensity was determined according to the account of each patient. The application occurred for 20 minutes at 20 minutes intervals until the parturient reach $8 \mathrm{~cm}$ cervix dilation or when the fourth technical applications were concluded.

The other group received intervention through the CRYO technique, using plastic bags with ice positioned in the region between T10 and L1. The ice bag was applied for 20 minutes with a 20 minutes interval until the mother reach $8 \mathrm{~cm}$ cervix dilation or when the fourth technical applications occurred. The time chosen for the application based on the physiological principle of cold. (20)

In both groups, the VAS was applied at the beginning, at the end and at each interval of the technique application. Patients have adopted a position of comfort during the application of the protocol and they were not oriented to remain in any position.

For the data analysis, descriptive statistics were used through mean, standard deviation and simple frequency to describe the sample characteristic. To identify the pain parameter, before and after intervention, descriptive statistics were used, using the median. Simple frequency was used to determine the pain location. The Mann-Whitney $U$ test was used to compare the data between the techniques, and the Wilcoxon test was used to compare the values before and after in the same group, with a significance level of 0.05 .

\section{RESULTS}

Twenty-four parturient were selected for the study. The characteristics of both groups were homogeneous regarding age, gestational age and initial and final cervix dilatation, as shown in table 1 . According to the human body map, 4 parturient of the TENS group and 8 of the CRYO group indicated pain in the lumbar region and low abdomen, the others indicated pain in the lumbar region only.

The following data refer to the values indicated by the pain scale in each period of application of the techniques, comparing the results of the TENS and CRYO techniques (Table 2).

After the first application of both techniques, it was observed that the median remained the same in both groups, 
Table 1 - Description of the sample according to age, gestational age, marital data and initial and final dilation of care.

\begin{tabular}{lcc}
\hline \multicolumn{1}{c}{ Characteristics } & TENS & \multicolumn{1}{c}{ CRYO } \\
\hline Age in years & $23.45( \pm 5.18)$ & $20.76( \pm 2.58)$ \\
Gestational age in weeks & $40( \pm 1.36)$ & $39( \pm 1.30)$ \\
Married & 8 & 5 \\
Common-law marriage & 3 & 8 \\
Initial cervix dilatation (mean) & $4.18( \pm 0.40)$ & $4.23( \pm 0.41)$ \\
Final cervix dilatation (mean) & $6.27( \pm 1.61)$ & $5.93( \pm 1.18)$ \\
\hline
\end{tabular}

Table 2 - Comparison of VAS median in patients of the TENS group (VAS T) and CRYO group (VAS C)

\begin{tabular}{cccccc}
\hline $\begin{array}{c}\text { Evaluation } \\
\text { period }\end{array}$ & VAS T & VAS C & $p^{*}$ & $\mathbf{n ~ T}$ & $\mathbf{n ~ C}$ \\
\hline 1A & 8 & 8 & 0.246 & 11 & 13 \\
1B & 7 & 7 & 0.213 & 11 & 13 \\
2A & 7 & 6 & $0.034^{*}$ & 10 & 11 \\
2B & 9 & 8 & 0.141 & 10 & 11 \\
3A & 7 & 7 & 0.451 & 8 & 9 \\
3B & 7.5 & 8 & 0.330 & 8 & 9 \\
4A & 6.5 & 8 & 0.262 & 6 & 9 \\
4B & 6.5 & 8 & 0.111 & 6 & 9 \\
Final & 5.5 & 8 & 0.287 & 9 & 11 \\
\hline
\end{tabular}

* Mann Whitney U test - * $p \leq 0.05$

Legend: $1 \mathrm{~A}$ (initial evaluation before the first intervention); $1 \mathrm{~B}$ (evaluation after the first intervention); $2 \mathrm{~A}$ (evaluation before the second intervention); $2 \mathrm{~B}$ (evaluation after the second intervention); $3 \mathrm{~A}$ (evaluation before the third intervention); $3 \mathrm{~B}$ (evaluation after the third intervention); $4 \mathrm{~A}$ (evaluation before the fourth intervention); $4 \mathrm{~B}$ (evaluation after the fourth intervention); $\mathrm{nT}$ (total number of patients who received TENS technique in each intervention phase); $\mathrm{nC}$ (total number of patients who received the CRYO technical intervention in each phase).

Table 3 - Comparison of median values of VAS among the steps of applying the technique in participants underwent TENS

\begin{tabular}{ccc}
\hline Evaluation & Median & $\boldsymbol{p}^{*}$ \\
\hline 1A & 8 & $0.002^{*}$ \\
1B & 7 & \\
2A & 7 & 0.067 \\
2B & 9 & \\
3A & 7 & 0.055 \\
3B & 7.5 & \\
4A & 6.5 & 0.113 \\
4B & 6.5 & \\
Initial & 8 & 0.219 \\
Final & 5.5 &
\end{tabular}

* Wilcoxon Test - *p $\leq 0.05$

Legend: $1 \mathrm{~A}$ (first evaluation before the intervention); $1 \mathrm{~B}$ (evaluation after the first intervention); $2 \mathrm{~A}$ (evaluation before the second intervention); $2 \mathrm{~B}$ (evaluation after the second intervention); $3 \mathrm{~A}$ (evaluation before the third intervention); $3 \mathrm{~B}$ (evaluation after the third intervention); $4 \mathrm{~A}$ (evaluation before the fourth intervention); $4 \mathrm{~B}$ (evaluation after the fourth intervention). and there was no significant difference between the therapies. However, at the beginning of the second intervention, there was a significant difference in the pain perception of the CRYO group in relation to the TENS group, and the participants reported a lower level of pain in the CRYO group. In the other applications, there were no significant differences between the two application techniques. There was a relative reduction of the pain scale values in the TENS group and with the CRYO group there was the maintenance of the values indicated by the participants after the second application.

The following table 3 shows the comparison of values before and after the application of TENS technique in each period of evaluation.

In the comparison of values before and after application, a significant result was obtained only after the first application of the technique. During the other applications, it may be analyzed that there was a slight modification of the VAS value, and at the end of the second application there was an increase in value. However, the observed value at the end of all applications was inferior to the original, but without significant difference.

The data in table 4 indicate the comparison of values before and after application of the CRYO technique.

In relation to the CRYO group, the reduction of pain during the first application of the technique was statistically significant. In the second application, there was a significant increase in pain. After the third application, there was the maintenance of median VAS value. Regarding the 11 participants in the TENS group, 8 received oxytocin administration, and 13 of the CRYO group, only 5 received it. Even with a lower number of parturient who received oxytocin in the CRYO group, there was a high VAS value during the applications, however without

Table 4 - Comparison of median values of VAS among the steps of applying the technique in participants underwent CRYO

\begin{tabular}{ccc}
\hline Evaluation & Median & $\boldsymbol{p}^{*}$ \\
\hline 1A & 8 & $0.008^{*}$ \\
1B & 7 & \\
2A & 6 & $0.008^{*}$ \\
2B & 8 & \\
3A & 7 & 0.341 \\
3B & 8 & \\
4A & 8 & 0.051 \\
4B & 8 & \\
Initial & 8 & 0.139 \\
Final & 8 &
\end{tabular}

* Wilcoxon Test - *p $\leq 0.05$

Legend: $1 A$ (first evaluation before the intervention); $1 B$ (evaluation after the first intervention); $2 \mathrm{~A}$ (evaluation before the second intervention); $2 \mathrm{~B}$ (evaluation after the second intervention); $3 \mathrm{~A}$ (evaluation before the third intervention); $3 \mathrm{~B}$ (evaluation after the third intervention); $4 \mathrm{~A}$ (evaluation before the fourth intervention); $4 \mathrm{~B}$ (evaluation after the fourth intervention). 
changing the value comparing the initial and final VAS. After the third application of the techniques, some patients had already reached uterine dilation of $8 \mathrm{~cm}$, therefore they did not continue with the treatment. In addition, three patients did not complete the study, two of which performed only the first application of the technique and one performed two applications.

\section{DISCUSSION}

The present study demonstrated that TENS and CRYO have a positive effect on pain during labor stages. There was no significant difference when compared the effect of the two techniques, therefore both may be used with good results.

There are still few studies associating TENS to pain relief during labor compared to other studies which prove its efficacy in other groups of patients. A study analyzed the combination of the two techniques (TENS/CRYO), as the study by Kanlayanaphotporn and Janwantanakul ${ }^{(21)}$ which confirmed the actual effectiveness of both in the reduction of pains of patients in different clinical conditions, especially postoperative.

The application of TENS does not seem to interfere with the duration of labor, incidence of cesarean section or instrumental delivery, nor causes adverse effects on the fetus ${ }^{(1)}$ and the technique used in order to facilitate analgesia process during childbirth and postpartum while minimizing discomfort and complications to the patients. (22) Possible complications and discomforts were not evaluated in this study, however indirectly it was observed that TENS did not bring any harm to the mother and baby.

As observed in the described results, the TENS technique proved to be effective in pain relief after the first application (initial phase), thus indicating that analgesia techniques should be applied in the early stages of labor. That evidence confirms the study by Chao et al, (5) in which used the TENS and placebo TENS, and their results showed pain relief also during the initial phase of labor and good acceptance by the participants regarding the adherence of the technique.

van Der Spank et al ${ }^{(23)}$ have also obtained good results using TENS during labor. The aim of the study was to determine the efficacy of TENS in pain relief during labor as well as its influence on the incidence of requests for epidural analgesia. The VAS scores in the group which received the technique was smaller, however there was no difference between the experimental group and control group with respect to the additional analgesia techniques requirement.

Orange et al (1) conducted a randomized clinical trial in order to determine the maternal and neonatal outcomes according to the application of TENS before the installation of the analgesia combined technique. The study establish that the application of TENS was effective in delaying the installation of combined anesthesia by maintaining analgesia longer than the control group. Ratna and Rekha ${ }^{(22)}$ used TENS in order to compare it to the use of Tramadal in labor progress and pain relief. Regarding the group which receive TENS, $80 \%$ achieved pain relief. The protocol used by the authors confirms the present study, however a fact which differs from this study is that during the uterine contraction the intensity of the device was increased, providing more stimulus and favoring a relief sense.

The CRYO was also more effective in relieving pain in the initial stage of labor, only during the first application, wherein the second application there was increased pain and after maintenance of its analgesic characteristic. This benefit may be explained according to the physiological principles of ice. Tyhopkins et al ${ }^{(24)}$ report that the ice, in addition to reducing the overall speed of nerve conduction, muscle spasm and pain, it also has a definite effect blocking and slowing sensory fibers.

The study conducted by Dehcheshmeh and Rafiei ${ }^{(25)}$ examined the effects of ice massage to relieve pain during delivery. At the beginning of the active phase $(4 \mathrm{~cm}$ of cervix dilatation) and before and after each intervention (dilatations 4, 6 and $8 \mathrm{~cm}$ ), labor pain intensities were measured using VAS. The test results showed that the reduction in pain intensity during the time intervals were statistically significant $(P=$ 0.001) and therefore cryotherapy was effective for relief of pain during labor. ${ }^{(25)}$

The cooling causes an increase in the duration of the sensory nerves action potential, and consequently an increase of the refractory period, decreasing the number of fibers which will depolarize, with a decrease in the impulse transmission frequency and a decrease of the pain sensitivity. ${ }^{(24)}$ Deceleration of nerve conduction velocity is the most likely mechanism for analgesic response to cold. The cold depresses the excitability of free nerve endings and peripheral nerve fibers which are responsible for the pain threshold, the decrease of the inflammatory mediator release which induce pain is one of the ice effects. ${ }^{(20,24)}$ The analgesia may be perceived seven to ten minutes of application, in most patients, and the effect of cold on the driving speed may last up to 30 minutes after application. ${ }^{(20,24)}$ Therefore, it is possible to be said that the first CRYO application that principle was demonstrated, since significant pain reduction was observed.

The median in the VAS results indicated that there was pain relief in the application of TENS, however this difference was not significant compared to CRYO group. This result may also have been influenced by the fact that the oxytocin was administered in 8 out of 11 patients who received the TENS technique. Oxytocin is a hormone which enhances uterine contractions making them strong and coordinated until completion of the delivery, in that as a release increases, the greater is the sensation of pain by the patient. ${ }^{(26)}$ In addition to the natural production, in many obstetrical centers, it is common the use of synthetic oxytocin, which aims to trigger and accelerate labor, so painful sensations may be triggered early. 
It is noteworthy that in the TENS group the value of the final VAS was lower in comparison with the initial and there were more patients who received oxytocin compared to the CRYO group. Thus, it is possible to observe that the TENS group had beneficial effects on pain relief during labor, even with a greater number of patients under oxytocin effect. As the intervention protocol was being performed, after the second application of the techniques, some parturient had already reached $8 \mathrm{~cm}$ cervix dilatation, and the treatment protocols had ceased. This fact may have influenced the results analysis after the third application of the techniques. As observed in the two intervention groups, the analgesic process occurred at the beginning of the applications. However, in the CRYO group, the value of VAS was maintained after the third application.

The results could also be influenced by the physiological process of delivery. The increase in cervix dilation is gradual, as it increases, so uterine contractions and pain sensation becomes more evident. ${ }^{(19,27)}$ According to Guyton and Hall, (27) delivery may be defined as a sequence of coordinates and involuntary uterine contractions which result in the progressive cervix dilation, leading to the expulsion of the fetus. Considering that at the beginning of labor the contractions are not as effective or rhythmic, if the alternative techniques for pain relief are applied at this stage there is a greater tendency to maintain the analgesic process, favoring the parturient to support the final phases in which the contractions become more painful, effective and rhythmic. ${ }^{(26)}$ That situation was observed in the present study in the TENS group.

\section{CONCLUSION}

The present research demonstrated that the CRYO and TENS techniques were effective in relieving pain during the first moment of the labor dilation phase. There was no significant difference when comparing the two techniques. However, TENS demonstrated a reduction of the initial and final pain values according to VAS. Although no significant reduction, the reduction may indicate some benefit in relieving pain during labor. Unlike CRYO, where the initial and final values remained the same, indicating that ice may provide maintenance of the pain.

Some considerations for future studies should be reported. It is suggested a study with larger sample and with greater balance among groups. It is also suggested a new study which includes this intervention protocol, however with a control group. Another factor which could be included in the protocol is the intensity adjustment according to the uterine contraction.

\section{AUTHORS' CONTRIBUTIONS}

MMR: design and research design, data collection, analysis and interpretation of data, drafting of the manuscript; GJSH: design and research design, analysis and interpretation of data, statistical analysis, drafting of the manuscript; KMS: analysis and interpretation of data, drafting of the manuscript; SCTL: design and research design, critical review of the manuscript as the important intellectual content; CML: design and research design, critical review of the manuscript as the important intellectual content; TR: critical review of the manuscript as the important intellectual content.

\section{CONFLICT OF INTEREST}

The authors declare no conflict of interest.

\section{AUTHOR DETAILS}

2Department of Physiotherapy, Universidade do Estado de Santa Catarina (UDESC), Florianópolis, SC, Brazil

\section{REFERENCES}

1. Orange FA, Amorim MMR, Lima L. Uso da eletroestimulação transcutânea para alívio da dor durante o trabalho de parto em uma Maternidadeescola: ensaio clínico controlado. Rev Bras Ginecol Obstet, 2003; 25(1): 45-52.

2. Davim RMB, Torres GV, Melo ES. Estratégias não farmacológicas no alívio da dor durante o trabalho de parto: pré-teste de um instrumento. Rev. Latino-Am. Enfermagem. 2007; 15(6): 1-7.

3. Lowe NK. The nature of labor pain. Am J Obstet Gynecol. 2002; 186(5): 16-24.

4. Pimenta CAM, Teixeira, MJ. Questionário de dor McGill: proposta de adaptação para a língua portuguesa. Rev Esc Enferm. 1996; 30(3): 473483.

5. Chao AS, et al. Pain relief by applying transcutaneous electrical nerve stimulation (tens) on acupuncture points during the first stage of labor: A randomized double-blind placebo-controlled. Pain; 2007; 127: 214-220.

6. Pitangui AC, Araújo RC, Bezerra MJ, Ribeiro CO, Nakano AM. Low and highfrequency TENS in post-episiotomy pain relief: a randomized, double-blind clinical trial. Braz J Phys Ther. 2014;18(1):72-8.

7. Allaire, A.D. Complementary and alternative medicine in the labor and delivery suite. Clin Obstet Gynecol. 2001; 44(1): 681-91.

8. Haynes JM. Randomized controlled trial of cryoanalgesia (ice bag) to reduce pain associated with arterial puncture. Respir Care. 2015; 60(1):15 .

9. Nugraha B, Günther JT, Rawert H, Siegert R, Gutenbrunner C. Effects of whole body cryo-chamber therapy on pain in patients with chronic low back pain: a prospective double blind randomised controlled trial. Eur J Phys Rehabil Med. 2015;51(2):143-8.

10. Santuzzi CH, Gonçalves WLS, Rocha SS et al. Efeitos da crioterapia, estimulação elétrica transcutânea e da sua associação na atividade elétrica do nervo femoral em ratos. Rev. Bras. Fisioter. 2008; 12(6): 441-446.

11. Bergeron-Vézina K, Corriveau H, Martel M, Harvey MP, Léonard G. Highand low-frequency transcutaneous electrical nerve stimulation does not reduce experimental pain in elderly individuals. Pain. 2015; 156(10):20939.

12. Park C, Choi JB, Lee YS, Chang HS, Shin CS, Kim S, et al. The effect of intra-operative transcutaneous electrical nerve stimulation on posterior neck pain following thyroidectomy. Anaesthesia. 2015;70(4):434-9. doi: 10.1111/anae.12933.

13. Straube A, Ellrich J, Eren O, Blum B, Ruscheweyh R. Treatment of chronic migraine with transcutaneous stimulation of the auricular branch of the vagal nerve (auricular t-VNS): a randomized, monocentric clinical trial. J Headache Pain. 2015;16(1):543.

14. Ferreira CHJ, Beleza ACS. Abordagem fisioterapêutica na dor pósoperatória: a eletroestimulação nervosa transcutânea (ENT). Rev. Col. Bras. Cir. 2007; 34(2) 127-130.

15. Miquelutti MA, Cecatti JG, Morais SS, Makuch MY. Posição vertical durante o trabalho de parto: dor e satisfação. Rev. Bras. Saúde Mater. Infant. 2009; 9(4): 393-398.

16. Marques AP, Peccin SM. A Pesquisa em fisioterapia: a prática baseada em evidências e modelos de estudo. Fisioter. Pesqui. 2005; 11(1): 43-48. 
17. Niven CA, Murphy-black T. Memory for labor pain: a review of the literature. Birth. 2000; 27(4): 244-253.

18. Pimenta CAM, Teixeira MJ. Questionário de dor McGill: proposta de adaptação para a língua portuguesa. Rev Esc Enferm. 1996; 30(3) 473-83.

19. Melo de Paula G, Molinero de Paula VR, Dias RO, Mattei K. Estimulação Elétrica Nervosa Transcutânea (TENS) no pós-operatório de cesariana. Rev. Bras. de Fisioter. 2006; 10(2): 219-224.

20. Hubbard TJ, Aronson SL, Denegar CR. Does Cryotherapy Hasten Return to Participation? A Systematic Review. J Athl Train. 2004; 39(1): 88-94.

21. Kanlayanaphotporn R, Janwantanakul P. Comparison of skin surface temperature during the application of various cryotherapy modalities. Arch Phys Med Rehabil. 2005; 86(7): 1411-5.

22. Ratna T, Rekha P. Comparative Study of Transcutaneous Electrical Nerve Stimulation (TENS) and Tramadol Hydrochloride for Pain Relief in Labor. J Obstet Gynaecol India. 2004; 54(4): 346-350.
23. Van der Spank JT, Cambier DC, De Paepe HM. Pain relief in labour by transcutaneous electrical nerve stimulation (TENS). Archives of Gynecol and Obstetrics. 2000; 264(10): 131-136.

24. Tyhopkins J. Cryotherapy and Transcutaneous Electric Neuromuscular Stimulation Decrease Arthrogenic Muscle Inhibition of the Vastus Medialis After Knee Joint Effusion. J Athl Train. 2001; 37(1): 25-31.

25. Dehcheshmeh FS, Rafiei H. Complementary and alternative therapies to relieve labor pain: A comparative study between music therapy and Hoku point ice massage. Complement Ther Clin Pract. 2015;21(4):229-32.

26. Rezende M. Obstetrícia Fundamental. 9.ed. Rio de Janeiro: Guanabara Koogan, 2003.

27. Guyton AC, Hall JE. Tratado de Fisiologia Médica. 11a ed. Rio de Janeiro, Elsevier Ed., 2006. 